\title{
TU/e EN⿴HONE

\section{Strong plasmon enhancement of the saturation photon count rate of single molecules}

\section{Citation for published version (APA):}

Wang, Y., Horáček, M., \& Zijlstra, P. (2020). Strong plasmon enhancement of the saturation photon count rate of single molecules. Journal of Physical Chemistry Letters, 11(5), 1962-1969.

https://doi.org/10.1021/acs.jpclett.0c00155

\section{Document license:}

CC BY-NC-ND

DOI:

10.1021/acs.jpclett.0c00155

Document status and date:

Published: 05/03/2020

\section{Document Version:}

Publisher's PDF, also known as Version of Record (includes final page, issue and volume numbers)

\section{Please check the document version of this publication:}

- A submitted manuscript is the version of the article upon submission and before peer-review. There can be important differences between the submitted version and the official published version of record. People interested in the research are advised to contact the author for the final version of the publication, or visit the $\mathrm{DOI}$ to the publisher's website.

- The final author version and the galley proof are versions of the publication after peer review.

- The final published version features the final layout of the paper including the volume, issue and page numbers.

Link to publication

\section{General rights}

Copyright and moral rights for the publications made accessible in the public portal are retained by the authors and/or other copyright owners and it is a condition of accessing publications that users recognise and abide by the legal requirements associated with these rights.

- Users may download and print one copy of any publication from the public portal for the purpose of private study or research.

- You may not further distribute the material or use it for any profit-making activity or commercial gain

- You may freely distribute the URL identifying the publication in the public portal.

If the publication is distributed under the terms of Article $25 \mathrm{fa}$ of the Dutch Copyright Act, indicated by the "Taverne" license above, please follow below link for the End User Agreement:

www.tue.nl/taverne

Take down policy

If you believe that this document breaches copyright please contact us at:

openaccess@tue.nl

providing details and we will investigate your claim. 


\title{
Strong Plasmon Enhancement of the Saturation Photon Count Rate of Single Molecules
}

\author{
Yuyang Wang, Matěj Horáček, and Peter Zijlstra*
}

Cite This: J. Phys. Chem. Lett. 2020, 11, 1962-1969

Read Online

ABSTRACT: Plasmon resonances have appeared as a promising method to boost the fluorescence intensity of single emitters. However, because research has focused on the enhancement at low excitation intensity, little is known about plasmon-fluorophore coupling near the point where the dye saturates. Here we study plasmon-enhanced fluorescence at a broad range of excitation intensities up to saturation. We adopt a novel DNA-mediated approach wherein dynamic single-molecule binding provides a controlled particle-fluorophore spacing, and dynamic rebinding circumvents artifacts due to photobleaching. We find that near saturation the maximum photon count rate is enhanced by more than 2 orders of magnitude at the optimal particle-fluorophore spacing, even for a dye with a high intrinsic quantum yield. We compare our results to a numerical model taking into account dye saturation. These experiments provide design rules to maximize the photon output of single emitters, which will open the door to studying fast dynamics in real time using single-molecule fluorescence.

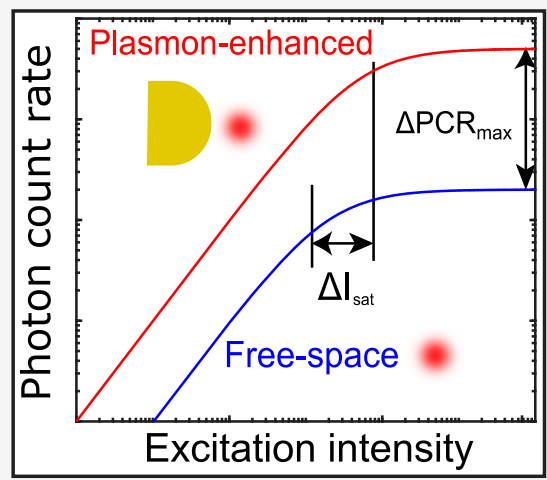

$S_{\mathrm{n}}^{\mathrm{in}}$ ingle-molecule fluorescence is a powerful technique to gain molecular-level insight into biological processes. Singlemolecule studies directly reveal molecular properties and their underlying statistics, instead of ensemble observables that obscure underlying distributions and nonsynchronized processes. ${ }^{1,2}$ Binding kinetics, for example, can be visualized using fluorescently labeled ligands such as DNA or proteins that dynamically bind and unbind to target molecules. ${ }^{3,4}$ Singleenzyme kinetics have also been revealed by means of fluorogenic substrates, in which single catalytic turnover events can be recorded. 5 Conformational dynamics have been probed based on fluorescence quenching, fluorescence anisotropy and lifetime, ${ }^{7}$ and Föster resonance energy transfer (FRET), allowing the fundamental investigation of structurefunction relationships. ${ }^{8-10}$ Indeed, single-molecule sensitivity impacts applications and has enabled crucial advances in medical diagnostics and biosensing, wherein, for example, continuous monitoring of biomolecules and single-molecule sequencing have been demonstrated. ${ }^{11-14}$

The brightness, or detected photon count rate (PCR), of single fluorophores is essential in many applications but strongly depends on the electronic properties and associated excitation and decay channels of the fluorophore. It is ideal in the detection of biological process to use fluorescent dyes with high PCR and chemical stability to allow highly sensitive single-molecule measurements. However, the PCR of single organic dyes is limited to $10^{6}-10^{7}$ photons per second, of which typically only $1 \%$ reaches the detector because of limited collection efficiency. ${ }^{15-17}$ The maximum PCR is intrinsically limited by saturation, which is the result of the finite decay rate of the fluorophore from the excited state and the presence of long-lived triplet excited states. This limits the maximum excitation rate that can be achieved because one has to "wait" for decay back to the ground state. The limited brightness of single molecules limits the signal-to-noise ratio particularly at short integration times and has prohibited real-time singlemolecule studies at submillisecond time scales.

Plasmon resonances have appeared as a promising method to boost the fluorescence intensity of single emitters. ${ }^{18}$ Since Drexhage first demonstrated that the spontaneous emission rate of fluorescent molecules could be accelerated by coupling to the photonic modes of nearby metal surfaces, much effort has been spent to study the interactions of plasmonic nanostructures and fluorophores. ${ }^{19}$ Metal nanoparticles stand out with excellent optical properties and have been used to enhance single-molecule fluorescence. ${ }^{20,21}$ The origin of these fluorescence enhancements is the strong modification of the excitation and decay rates of a molecular dipole close to a particle. The excitation rate is locally increased because of the enhanced electromagnetic field in the vicinity of the particle surface, while the radiative and nonradiative decay rates are strongly modified because of the coupling of the molecular dipole moment to the plasmon modes of the particle. Both excitation and decay-rate modification are strongly dependent on the optical properties of the nanoparticle and the fluorophore.

Received: January 15, 2020

Accepted: February 19, 2020

Published: February 19, 2020 
Most research has focused on the quantification of enhancement factors, which indicate by what factor the total detected fluorescence intensity is enhanced when a fluorophore is brought in proximity to a metal particle. ${ }^{22-24}$ These studies have shown that the fluorescence of single weakly emitting molecules can be enhanced by thousands of times. $^{25-27}$ High enhancement factors at low excitation power have mostly been applied to perform single-molecule spectroscopy at high concentrations. ${ }^{28-31}$ However, a different class of applications involves real-time single-molecule fluorescence measurements of biomolecular dynamics, for which not the fluorescence enhancement but rather the absolute PCR matters. To achieve the highest PCR it is necessary to pump molecules close to or even above fluorescence saturation. ${ }^{32,33}$ However, little is known about the effect of plasmon-molecule coupling on the saturation dynamics of single dyes, and design rules to achieve maximum absolute photon count rates are not known.

Here we present a single-molecule study of plasmonenhanced fluorescence of a dye with a high intrinsic quantum yield that is pumped close to saturation. A particular concern is preventing artifacts due to photobleaching, for which we adopt an approach based on low-affinity DNA interactions resulting in controlled residence times of the fluorophore near the particle, continuous refreshment of the dye by rebinding, and a well-controlled spacing between particle and fluorophore. We measure the PCR as a function of excitation power, from which we derive the $\mathrm{PCR}_{\max }$ and saturation intensity $\left(I_{\text {sat }}\right)$. The PCR is strongly modified because of plasmon-fluorophore coupling, while we find thatthe $\mathrm{PCR}_{\max }$ is enhanced by more than 2 orders of magnitude at $\sim 1.5 \mathrm{~nm}$ from the particle surface. The experimental findings are in good agreement with numerical simulations that include saturation. These experiments provide mechanistic insight and design rules to optimize the absolute photon output of single emitters. This will open the door to studying microsecond dynamics in real time, e.g., DNA hairpin dynamics and transition paths in protein folding. ${ }^{34-37}$

To explain the origin of fluorescence saturation we employ a simplified model that represents the dye as a three-level system as in Figure 1a, in which the ground state $S_{0}$, the first excited singlet state $S_{1}$ and the triplet state $T_{1}$ are shown. Upon illumination, the molecule is excited from $S_{0}$ to $S_{1}$ with a certain excitation rate $\gamma_{\text {exc }}=\sigma_{\mathrm{abs}} \frac{I_{\text {exc }}}{h \nu}\left[\mathrm{s}^{-1}\right]$, where $\sigma_{\mathrm{abs}}$ is the
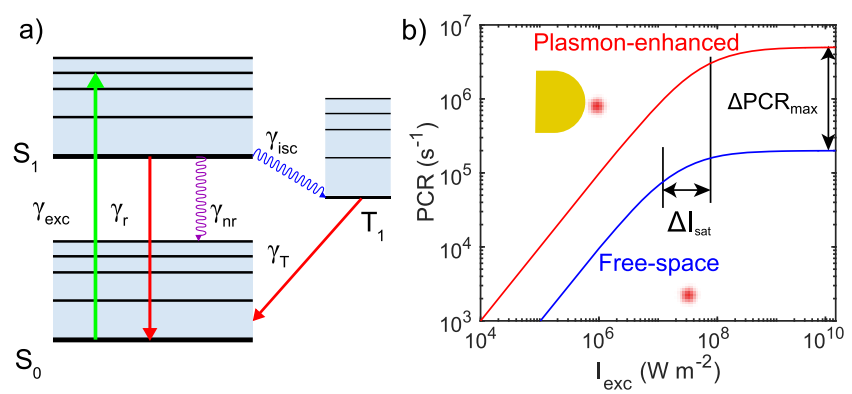

Figure 1. Photophysics of a single emitter modified by a plasmonic nanoantenna. (a) A three-level Jablonski diagram for a single fluorescent molecule. (b) Cartoon of saturation curves of an emitter in free space (blue) and in the vicinity of a plasmonic nanoantenna. Both the saturation intensity $\left(I_{\text {sat }}\right)$ and the maximum photon count rate $\left(\mathrm{PCR}_{\max }\right)$ are modified. Note here that this figure is for illustrative purposes and is not a simulation result. absorption cross section of the molecule $\left[\mathrm{m}^{2}\right]$ and $I_{\text {exc }}$ is the illumination power density $\left[\mathrm{W} \mathrm{m}^{-2}\right]$. The molecule relaxes from $S_{1}$ to $S_{0}$ emitting photons with a radiative decay rate $\gamma_{\mathrm{r}}$ or nonradiatively with a nonradiative decay rate $\gamma_{\mathrm{nr}}$. The molecule may transition from $S_{1}$ to $T_{1}$ with an intersystem-crossing rate of $\gamma_{\text {isc }}$ and decays from $T_{1}$ to ground state $S_{0}$ with a triplet decay rate $\gamma_{\mathrm{T}}$. The total decay rate $\gamma_{\text {tot }}=\gamma_{\mathrm{r}}+\gamma_{\mathrm{nr}}+\gamma_{\mathrm{isc}}$ is related to the fluorescence lifetime, while the quantum yield $\phi=\frac{\gamma_{\mathrm{r}}}{\gamma_{\text {tot }}}$ dictates the fraction of excitations that result in photon emission. The PCR of a fluorescent molecule is then given by

$$
\mathrm{PCR}=\eta_{\mathrm{col}} \frac{\sigma_{\mathrm{abs}}}{h \nu} \phi \frac{I_{\mathrm{exc}} I_{\mathrm{sat}}}{I_{\mathrm{exc}}+I_{\mathrm{sat}}}
$$

where $\eta_{\text {col }}$ is the collection efficiency of the setup as a whole, and the saturation intensity is written as ${ }^{38}$

$$
I_{\mathrm{sat}}=\frac{h \nu}{\sigma_{\mathrm{abs}}} \frac{\gamma_{\mathrm{tot}}}{1+\gamma_{\mathrm{isc}} / \gamma_{\mathrm{T}}}
$$

Equation 1 indicates the nonlinear dependence of PCR at excitation rates around and above $I_{\text {sat }}$, whereas eq 2 indicates that $I_{\text {sat }}$ is dictated by the total decay rate and the triplet dynamics of the molecule. As conceptually shown in Figure $1 \mathrm{~b}$, with increasing excitation power density, the PCR of both freespace and plasmon-enhanced single molecules lose linearity and approach a plateau $\mathrm{PCR}_{\max }$ that is expressed as $\mathrm{PCR}_{\text {max }}=\eta_{\text {col }} \frac{\sigma_{\text {abs }}}{h \nu} \phi I_{\text {sat }}$ because $\lim _{I_{\text {exc }} \rightarrow \infty} \frac{I_{\text {exc }} I_{\text {sat }}}{I_{\text {exc }}+I_{\text {sat }}}=I_{\text {sat }}$. In the presence of a nanoparticle, both the emission and excitation rates in eq 1 are strongly modified. First, the excitation rate in the vicinity of a single nanoparticle is strongly enhanced because of the concentrated electromagnetic field induced by the plasmon resonance. Second, both $\gamma_{\mathrm{r}}$ and $\gamma_{\mathrm{nr}}$ are strongly increased because of coupling with plasmon modes in the nanoparticle. We first assume that triplet dynamics is not affected by plasmon coupling, but we will return to this point later.

The net effect of these rate modifications is a modification of the saturation dynamics as illustrated in Figure $1 b$, showing that the PCR is enhanced at all power densities because of the local field enhancement. Notably, also $\mathrm{PCR}_{\max }$ is significantly enhanced because of a modified $I_{\text {sat }}$. The plasmonic modification of the photophysics strongly depends on the optical properties of both the nanoparticle and the fluorophore, and also their relative position and orientation. The effect of nanostructures on fluorescence saturation was first experimentally investigated by Ebbesen et al. to show that nanoapertures enhance the PCR of diffusing dyes on an ensemble-averaged level. ${ }^{39}$ More recently van Hulst et al. reported that an up to 100 -fold decrease in excitation power was required to reach $I_{\text {sat }}$ for single light-harvesting complexes coupled to single gold nanoparticles because of near-field enhancement. They also observed a 50-fold enhancement of $\mathrm{PCR}_{\max }$ due to modification of the emitter's decay rates. ${ }^{40}$

We numerically evaluate the modifications of the radiative and nonradiative rates using the boundary element method (BEM) ${ }^{41}$ and by combining the numerical simulation results with the three-level photophysical model as in eq 1 we calculate the modification of the saturation dynamics for particles of various dimensions. Near saturation the $\mathrm{PCR}_{\max }$ is proportional to the radiative rate enhancement, namely 

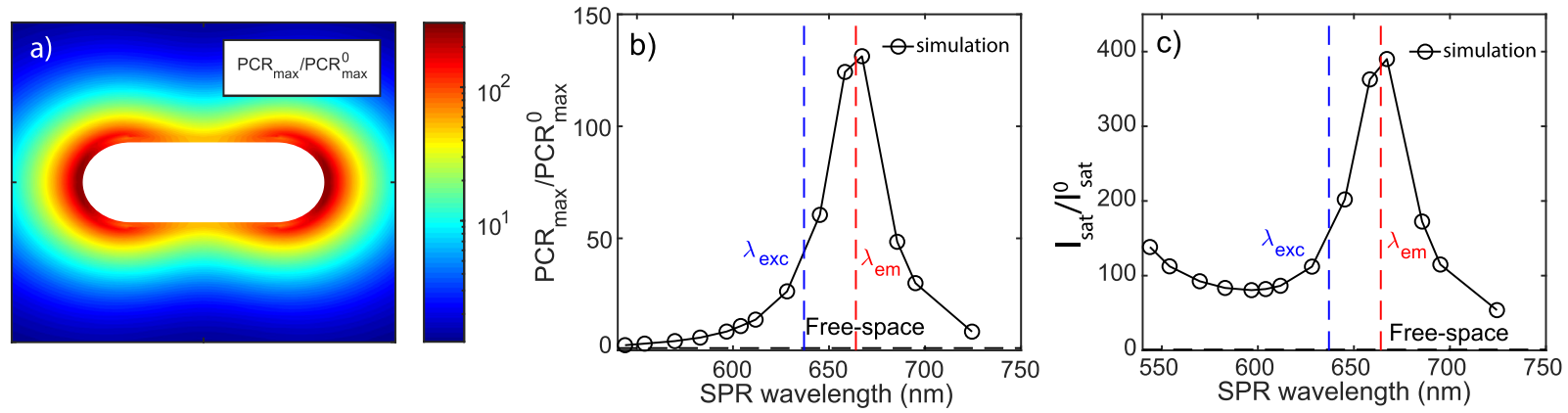

Figure 2. Numerical calculations of photophysical modifications in the vicinity of a single gold nanorod with a size of $63 \mathrm{~nm} \times 25 \mathrm{~nm}$. (a) PCR $\max$ modifications normalized to that of a free space emitter $\mathrm{PCR}_{\max }^{0}$, calculated with orientation-averaged radiative and total decay rate modifications as a function of fluorophore position. Fluorophores are assumed to be single-wavelength dipole emitters with a quantum yield of unity at $664 \mathrm{~nm}$ (resonant with the particle plasmon). (b) $\mathrm{PCR}_{\max }$ modification normalized by the value of a free space fluorophore $\mathrm{PCR}_{\max }^{0} \cdot \lambda_{\text {exc }}=637 \mathrm{~nm}$ and $\lambda_{\text {em }}$ $=664 \mathrm{~nm}$ indicate the excitation and emission wavelengths. The SPR wavelength of the particles was tuned by changing their length while keeping their diameter at $25 \mathrm{~nm}$. (c) $I_{\text {sat }}$ modification normalized by the saturation intensity of a free space fluorophore $I_{\text {sat }}^{0}$. For wavelength-dependent calculations, the dipole emitter is placed at $4 \mathrm{~nm}$ from the tip, and the results are orientation averaged.
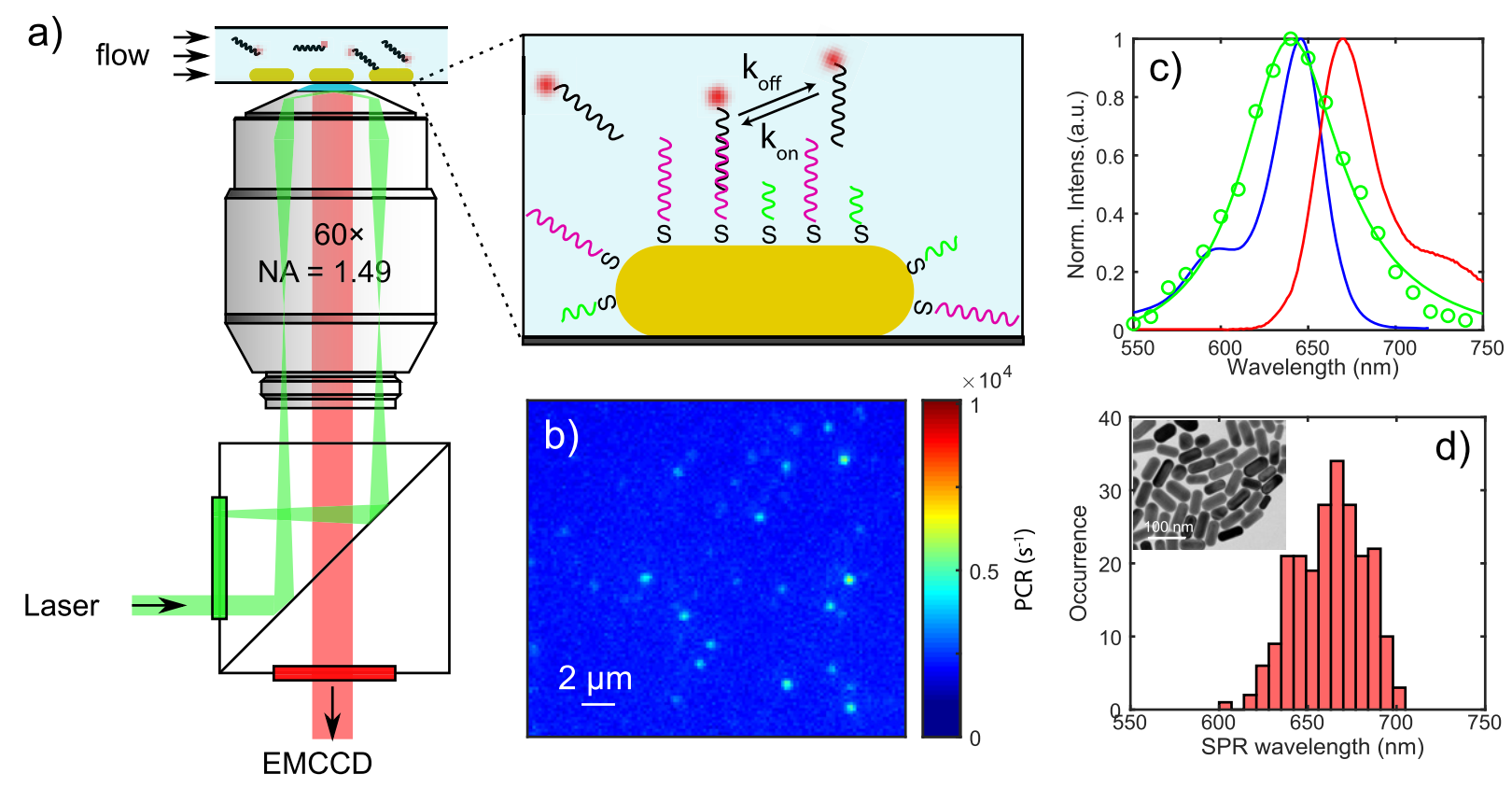

Figure 3. Single-molecule fluorescence microscopy and single-particle spectroscopy. (a) Schematic illustration of the setup for objective-type total internal reflection fluorescence (o-TIRF) microscopy, with the inset showing the surface functionalization and DNA binding on a single gold nanorod. (b) EMCCD image of immobilized DNA-funtionalized AuNRs immersed in imager solution, with diffraction-limited spots from the onephoton photoluminescence of single AuNRs. (c) Hyperspectral scattering spectrum of a typical single AuNR with a longitudinal SPR wavelength of $640 \mathrm{~nm}$ (green) and the ensemble absorption/emission spectra (blue and red) of ATTO $647 \mathrm{~N}$ in aqueous buffer. (d) The distribution of longitudinal SPR wavelengths measured by single-particle hyperspectral spectroscopy. The inset is a TEM image showinga dried droplet of suspension from which we obtained the size and geometry of the AuNRs.

$$
\frac{\mathrm{PCR}_{\max }}{\mathrm{PCR}_{\max }^{0}}=\frac{\phi}{\phi_{0}} \frac{I_{\mathrm{sat}}}{I_{\mathrm{sat}}^{0}}=\frac{\gamma_{\mathrm{r}}}{\gamma_{\mathrm{r}}^{0}} \frac{1+\gamma_{\mathrm{isc}}^{0} / \gamma_{\mathrm{T}}^{0}}{1+\gamma_{\mathrm{isc}} / \gamma_{\mathrm{T}}}
$$

where the superscript " 0 " indicates free-space values. Equation 3 indicates that the $\mathrm{PCR}_{\max }$ enhancement follows the position and wavelength dependence of the $\gamma_{\mathrm{r}}$ enhancement, as shown in Figure 2a. (Numerical simulation results for comparison can be found in the Supporting Information.) In Figure $2 b$ we show the $\mathrm{PCR}_{\max }$ modification normalized to free-space as a function of SPR wavelength. We find indeed that the maximum $\mathrm{PCR}_{\max }$ enhancement up to $\sim 150$ when the SPR wavelength of the particle coincides with the fluorophore's emission wavelength, whereas the enhancement is a factor of $3 \times$ lower when the SPR is resonant with the excitation wavelength. This is fundamentally different in the low-excitation regime where most other studies have been performed, where the enhancement is dominated by the excitation process. In Figure $2 \mathrm{c}$ we show the modification of $I_{\text {sat }}$ normalized to its free-space values. A maximum $I_{\text {sat }}$ increase of $\sim 400$ is found for a plasmon that is resonant with $\lambda_{\mathrm{em}}$ because of the strong wavelength dependence of the decay rates that again exhibit a maximum at $\lambda_{\mathrm{em}}$ as in eq 2 . This implies that substantially higher excitation power densities are needed to reach the saturation regime for a plasmon-coupled fluorophore, but this is alleviated by the strong near-field enhancement that results in dye saturation at acceptable laser powers.

To experimentally study $\mathrm{PCR}_{\max }$ and $I_{\text {sat }}$ we perform singlemolecule fluorescence microscopy on single gold nanorods. 

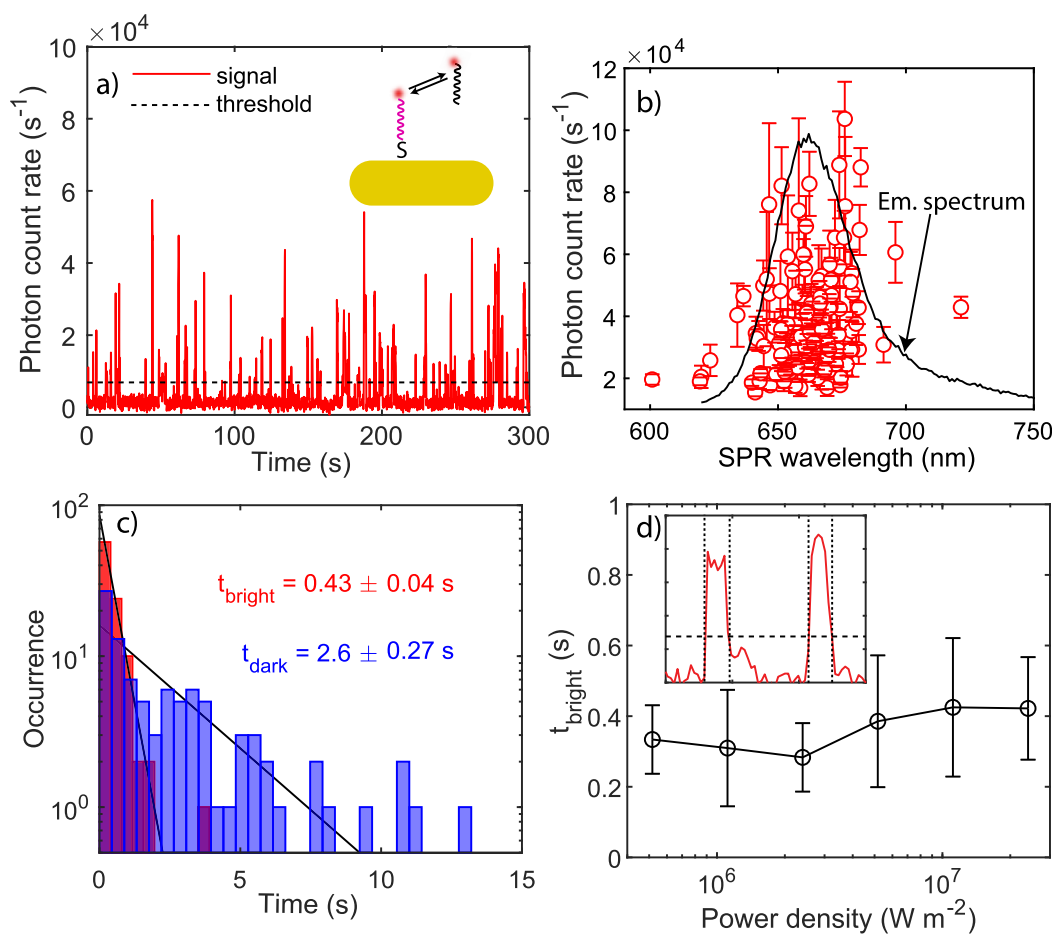

Figure 4. Fluorescence time traces and correlation with SPR wavelengths of single AuNRs. (a) Fluorescence time trace showing single imager strands binding to single AuNRs. This AuNR's SPR wavelength is determined to be $661 \mathrm{~nm}$. (b) SPR wavelengths of $\sim 130$ single AuNRs correlated with the mean photon count rates of the 10 brightest events. The error bars indicate the standard deviation of the count rates. (c) Dark and bright time distributions of imager binding. (d) Mean bright times as a function of power density. The error bars indicate the standard deviation of mean bright times collected from up to 100 single nanorods. The inset illustrates the determination of bright times from on a fluorescence time trace.

The heterogeneous size distribution of the colloidally synthesized gold nanorods allows us to study the SPR wavelength dependence in a single measurement. In Figure 3a we illustrate the detection of single particles and single fluorescent molecules based on an objective-type TIRF configuration. Gold nanorods are first spin-coated on a microscope coverslip, functionalized with a mixture of thiolated single-stranded docking strands and short $10 \mathrm{nt}$ antifouling strands, and mounted in a flow cell. Imager strands fluorescently labeled with ATTO647N with a 9 nt complementary sequence to the docking strands are injected into the flow cell under a continuous flow of $100 \mu \mathrm{L} / \mathrm{min}$. This results in controlled residence times of the fluorophore near the particle dictated by the affinity $(\sim 1 \mu \mathrm{M})$, continuous refreshment of the dye by rebinding, and a well-controlled spacing between particle and fluorophore.

In Figure $3 \mathrm{~b}$ we show the detected one-photon photoluminescence of single gold nanorods on the EMCCD camera under $637 \mathrm{~nm}$ laser excitation. Camera counts were converted to photon counts by measuring in the photon-counting mode of the EMCCD. The scattering spectra of each particle are measured with hyperspectral imaging under white light illumination in the same field of view. ${ }^{42}$ A typical scattering spectrum of a particle is shown in Figure 3c, from which the SPR wavelength is acquired after Lorentzian fitting. The particles exhibit a distribution of SPR wavelengths due to size nonuniformity inherited from colloidal synthesis which we exploit here to study plasmon-enhanced fluorescence as a function of spectral overlap between the fluorophore and the plasmon. In Figure 3d we show the distribution of longitudinal SPRs for $\sim 300$ single nanorods in a single field of view.
Upon filling the flow cell with imager solution, we observe fluorescent bursts appearing as point spread functions (PSFs) superposed on the constant one-photon photoluminscence of single gold nanorods. In Figure $4 \mathrm{a}$ we show the detected PCR from frame-by-frame summation of 9-by-9 pixels centered around a single gold nanorod, excited by a $637 \mathrm{~nm}$ s-polarized $\mathrm{CW}$ laser with a power density in the focal plane of $2 \times 10^{7} \mathrm{~W}$ $\mathrm{m}^{-2}$. The large variation in the fluorescence intensities from these bursts can be attributed to the random binding location of each imager. Because it is expected that the maximally enhanced bursts originate from binding events close to the tip of the nanorods, we average the PCR of the 10 strongest fluorescence events and correlate them with the SPR wavelength of the same particle as shown in Figure $4 \mathrm{~b}$. We find that the optimum PCR enhancement occurs near the emission peak of the dye. This indicates that the PCR enhancement is dominated by the modification of the emission process rather than the excitation process. Note that individual particles exhibit a different PCR even for the same plasmon wavelength. This is a result of the random orientation of the particles in the s-polarized excitation field formed by total internal reflection.

In order to separate the signals of binding events from background, we applied a threshold $(\sim 10 \times$ the background noise) to locate the timestamps of signals. The characteristic bright times $\left(t_{\text {bright }}\right)$ and dark times $\left(t_{\text {dark }}\right)$ of the transient binding events can be extracted from the time trajectory. $t_{\text {bright }}$ and $t_{\text {dark }}$ are related to the thermodynamic properties of the single-molecule DNA interaction and can be directly related to the (single-site) association rate $k_{\text {on }}=\frac{1}{t_{\text {dark }} c_{\text {imager }}}$ and the 
dissociation rate $k_{\text {off }}=\frac{1}{t_{\text {bright }}}$, with $c_{\text {imager }}$ being the imager concentration. ${ }^{43}$ In Figure $4 \mathrm{c}$ we show the extracted $t_{\text {bright }}$ and $t_{\text {dark }}$ histograms and fit single-exponential distributions for both. We find $t_{\text {bright }}=0.4 \pm 0.1 \mathrm{~s}$ and $t_{\text {dark }}=3 \pm 0.3 \mathrm{~s}$, in good agreement with previous reports where $9 \mathrm{bp}$ duplexes were used. ${ }^{43,44}$

Photobleaching of a fluorophore might occur via tripletmediated mechanisms ${ }^{45}$ and via higher-order excited states. ${ }^{46}$ We demonstrate experimentally that the effect of photobleaching is negligible in our experiments because of the refreshment of fluorophores by repeated DNA hybridization. The burst duration is thus controlled by the number of complementary nucleotides. This is evidenced by the fact that $t_{\text {bright }}$ is independent of laser power. In Figure $4 \mathrm{~d}$ we show the mean $t_{\text {bright }}$ as a function of power density, collected from time traces of $\sim 100$ single gold nanorods. We find that $t_{\text {bright }}$ is $0.4 \mathrm{~s}$ independent of power density, which confirms that any nonlinearity in power-dependent PCR is not caused by photobleaching. Fluorescence time traces and histograms of $t_{\text {bright }}$ distributions measured under different power densities can be found in the Supporting Information.

Near saturation, the PCR enhancement compared to freespace excitation becomes power-dependent. We therefore determine saturation curves by collecting time trajectories of fluorescence bursts under different excitation power densities ranging from $5.2 \times 10^{5}$ to $2.4 \times 10^{7} \mathrm{~W} \mathrm{~m}^{-2}$. The dynamic range of the power density series is limited by the laser spot size and excitation laser power (see the Supporting Information for more details). All the saturation curves are fitted with eq 1 yielding $\mathrm{PCR}_{\max }$ and $I_{\text {sat. }}$. In Figure 5 we show

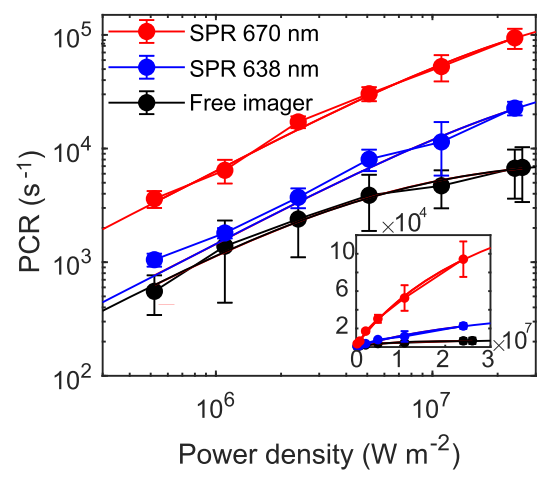

Figure 5. Saturation curves of plasmon-enhanced and nonenhanced single-molecule fluorescence. Inset shows the same saturation curves on a linear scale. The saturation intensity of free imagers is determined to be $I_{\text {sat }}^{0}=6.4 \times 10^{6} \mathrm{~W} \mathrm{~m}^{-2}$, and maximum PCR at saturation $\mathrm{PCR}_{\max }^{0}=8.3 \times 10^{3} \mathrm{~s}^{-1}$. For the particle with a SPR of 670 $\mathrm{nm}, \mathrm{PCR}_{\max }^{670}=2.3 \times 10^{5} \mathrm{~s}^{-1}$ and $I_{\text {sat }}^{670}=3.5 \times 10^{7} \mathrm{~W} \mathrm{~m}^{-2}$. For the particle with a SPR of $638 \mathrm{~nm}, \mathrm{PCR}_{\max }^{638}=5.9 \times 10^{4} \mathrm{~s}^{-1}$ and $I_{\text {sat }}^{638}=3.9$ $\times 10^{7} \mathrm{~W} \mathrm{~m}^{-2}$

the saturation curves of free-space fluorophores (see the Supporting Information for more data regarding the saturation dynamics without an antenna) and plasmon-coupled fluorophores for two gold nanorods with a longitudinal SPR of 670 and $638 \mathrm{~nm}$, resonant with the excitation and emission wavelengths, respectively. We clearly observe a nonlinear dependence of the detected intensity commensurate with fluorescence saturation. As stated above, this nonlinearity is a result of fluorescence saturation instead of photobleaching at increased power density. We find that both $\mathrm{PCR}_{\text {max }}$ and $I_{\text {sat }}$ are significantly modified, and both are more enhanced for $670 \mathrm{~nm}$ than $638 \mathrm{~nm}$ plasmon wavelengths.

We now investigate the modified $\mathrm{PCR}_{\max }$ and $I_{\text {sat }}$ for more particles to reveal the SPR wavelength dependencies. In Figure 6 we show the SPR wavelength-dependent $\mathrm{PCR}_{\max }$ and $I_{\text {sat }}$
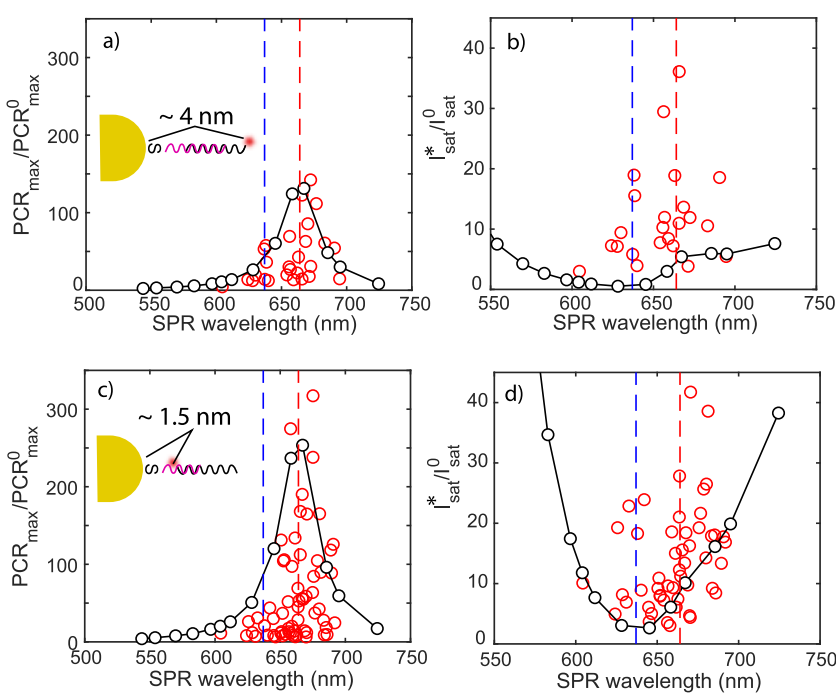

Figure 6. (a and b) Modified $\mathrm{PCR}_{\max }$ and $I_{\text {sat }}$ of plasmon-enhanced fluorescence for AuNRs coated with $30 \mathrm{nt}$ docking strands $(4 \mathrm{~nm}$ spacing) and ( $\mathrm{c}$ and d) for AuNRs coated with $15 \mathrm{nt}$ docking strands and near-end labeled imager ( $1.5 \mathrm{~nm}$ spacing). Note here that the simulated $I_{\text {sat }}^{*}$ is normalized to the near-field intensity at the position of the fluorophore to be able to display both the measured and simulated values on the same $y$-axis, i.e., $I_{\text {sat }}^{*}=\frac{I_{\text {sat }}}{|E|^{2} /\left|E_{0}\right|^{2}}$. In all graphs, red dots are experimental data points, and black dots are simulation results with lines as a guide to the eye. $R^{2}$ values are used to evaluate the fitting quality, and only curves with $R^{2}>0.995$ are plotted. See the Supporting Information for full simulation details including the decay rates used in the simulations.

which are obtained from the fits to the saturation curves. We employ here docking strands with different lengths to explore the dependence of the enhancement on the particlefluorophore spacing. In Figure $6 \mathrm{a}, \mathrm{b}$ we show the results for measurements in which $30 \mathrm{nt}$ docking strands are used to fully cover the gold nanorods, and imager strands with ATTO647N labeled at the far-end of the complementary sequence, leading to $\sim 4 \mathrm{~nm}$ time-averaged spacing between fluorophore and particle (see the Supporting Information for the calculation of the time-averaged spacing). Panels $\mathrm{c}$ and $\mathrm{d}$ of Figure 6 show the results for measurements with 15 nt docking strands and a flipped imager strand with ATTO647N at the near-end of the complementary region, shortening the time-averaged particlefluorophore spacing to $\sim 1.5 \mathrm{~nm}$.

In the above results, we find that the $\mathrm{PCR}_{\max }$ enhancement matches well with theory, with the calculated $\frac{\mathrm{PCR}_{\max }}{\mathrm{PCR}_{\max }^{0}}$ appearing as the upper limit in the experiments because these events originate from particles that are aligned with the excitation laser polarization. Particles with a longitudinal plasmon that is resonant with the emission of the dye enhance $\mathrm{PCR}_{\max }$ by nearly 300 fold, an effect dominated by the enhancement of the radiative rate as described before.

However, the predicted modification of $I_{\text {sat }}$ does not follow the measured modification. In Figure $6 \mathrm{~b}, \mathrm{~d}$ we show the correlation between the longitudinal SPR and $I_{\text {sat }}$, here plotted 
as the excitation laser power density required (in other words, Figure $2 \mathrm{f}$ divided by the orientation averaged wavelengthdependent near-field intensity at the location of the dye). We hypothesize that this could be due to plasmonic modification of triplet state dynamics. To understand this, it is useful to examine the dependence of $I_{\text {sat }}$ enhancement in the form

$$
\frac{I_{\text {sat }}}{I_{\text {sat }}^{0}}=\frac{\gamma_{\text {tot }}}{\gamma_{\text {tot }}^{0}} \frac{1+\gamma_{\mathrm{isc}}^{0} / \gamma_{\mathrm{T}}^{0}}{1+\gamma_{\mathrm{isc}} / \gamma_{\mathrm{T}}}
$$

In the simulations we have treated the term $\frac{1+\gamma_{\text {isc }}^{0} / \gamma_{\mathrm{T}}^{0}}{1+\gamma_{\mathrm{isc}} / \gamma_{\mathrm{T}}}$ appearing in both eqs 3 and 4 as unity, following Ebbesen et al. ${ }^{38}$ Considering the fact that the $\mathrm{PCR}_{\max }$ enhancement (and thus $\left.\gamma_{\mathrm{r}} / \gamma_{\mathrm{r}}^{0}\right)$ closely follows the prediction, this could indicate that plasmonic modification of triplet dynamics, or specifically the ratio $\gamma_{\text {isc }} / \gamma_{\mathrm{T}}$. An $I_{\text {sat }}$ enhancement up to 40 as we measured may therefore be the result of a $\sim 10$-fold modification of the ratio $\gamma_{\mathrm{isc}} / \gamma_{\mathrm{T}}$. Modification of triplet dynamics has been reported, but experimental studies are limited to a select number of cases $^{47-51}$ that indeed report modest modifications, in line with our observation. A quantitative investigation of triplet modifications requires a temporal resolution that is not accessible in our current camera-based setup but could be further investigated using, for example, fluorescence correlation spectroscopy (FCS). ${ }^{46,51,52}$

Our experiments indicate that the maximum photon count rate of a dye with an intrinsically high quantum yield can be enhanced by more than 100 -fold by plasmon coupling, enabling single-molecule studies with superior signal-to-noise ratio particularly for short integration times. We employed our numerical model to explore how $\mathrm{PCR}_{\max }$ and $I_{\text {sat }}$ depend on the size of the particle and photophysical properties of different types of emitters (see the Supporting Information). These simulations indicate that particles with a width in the range of 20-30 nm exhibit the highest $\mathrm{PCR}_{\max }$ enhancement. A reduced enhancement of $\mathrm{PCR}_{\max }$ for smaller particles is due to a reduced radiative efficiency of the plasmon, whereas larger particles suffer from line broadening due to radiation damping.

At a fixed particle size, the intrinsic radiative rate of the emitter then determines $\mathrm{PCR}_{\max }$. This indicates that certain classes of emitters might be more suitable to further push $\mathrm{PCR}_{\max }$. Comparing emitters with various intrinsic radiative rates (see the Supporting Information), we find that $\mathrm{PCR}_{\max }$ up to $10^{7} \mathrm{~s}^{-1}$ is feasible for emitters with $\gamma_{\mathrm{r}}^{0}=10^{9} \mathrm{~s}^{-1}$, corresponding to organic fluorophores, enzyme cofactors, and fluorescent proteins that typically have nanosecond fluorescence lifetimes. ${ }^{15}$ Semiconductor and carbon dots typically exhibit 1-10 ns lifetimes, whereas lanthanides and transition metal-ligand complexes with 100-1000 ns lifetimes are expected to yield $>100$ times lower plasmon-enhanced $\mathrm{PCR}_{\max }$ compared to organic fluorophores. ${ }^{53}$ Organic fluorophores as we used here thus provide the highest $\mathrm{PCR}_{\max }$, albeit at the expense of photostability compared to other emitters.

In summary, we presented a single-molecule study of plasmon-enhanced fluorescence of a dye with a high intrinsic quantum yield that is pumped close to saturation, where we focused on modification of the saturation dynamics by single metal nanoparticles using reversible single-molecule DNA hybridization. We have addressed the concern of photobleaching by employing low-affinity DNA interactions resulting in controlled residence times of the fluorophore near the particle, continuous refreshment of the dye by rebinding, and a well-controlled spacing between particle and fluorophore. We have measured the PCR as a function of excitation power, from which we derive the $\mathrm{PCR}_{\max }$ and saturation intensity $\left(I_{\text {sat }}\right)$. The PCR was strongly modified because of plasmon coupling with a $\mathrm{PCR}_{\max }$ that was enhanced by more than 2 orders of magnitude at $\sim 1.5 \mathrm{~nm}$ from the particle surface. The experimental findings were in good agreement with numerical simulations that include saturation. These experiments provide design rules to optimize the absolute photon output of single emitters. We envision that this will open the door to studying microsecond dynamics using real-time single-molecule fluorescence, e.g., DNA hairpin dynamics and transition paths in protein folding.

\section{ASSOCIATED CONTENT}

\section{Supporting Information}

The Supporting Information is available free of charge at https://pubs.acs.org/doi/10.1021/acs.jpclett.0c00155.

Sample preparation, single-molecule fluorescence microscopy and single-particle spectroscopy, calibration of TIRF excitation power density, free-space fluorescence saturation of single molecules, derivation of singlemolecule photon count rate, fluorescence timetraces as a function of power density and SPR wavelength, and numerical simulations (PDF)

\section{AUTHOR INFORMATION}

\section{Corresponding Author}

Peter Zijlstra - Department of Applied Physics and Institute for Complex Molecular Systems, Eindhoven University of Technology, 5600 MB Eindhoven, The Netherlands; ○ orcid.org/0000-0001-9804-2265; Email: p.zijlstra@tue.nl

\section{Authors}

Yuyang Wang - Department of Applied Physics and Institute for Complex Molecular Systems, Eindhoven University of Technology, 5600 MB Eindhoven, The Netherlands; (1) orcid.org/0000-0001-5175-8389

Matěj Horáček - Department of Applied Physics and Institute for Complex Molecular Systems, Eindhoven University of Technology, 5600 MB Eindhoven, The Netherlands

Complete contact information is available at: https://pubs.acs.org/10.1021/acs.jpclett.0c00155

\section{Notes}

The authors declare no competing financial interest.

\section{ACKNOWLEDGMENTS}

P.Z. and Y.W. acknowledge financial support from The Netherlands Organization for Scientific Research (NWO VIDI). We thank Marcel Verheijen for TEM imaging; Solliance and the Dutch province of Noord-Brabant are acknowledged for funding the TEM facility. This work is part of the research programme of the Foundation for Fundamental Research on Matter (FOM) which is financially supported by The Netherlands Organisation for Scientific Research (NWO). We thank Lex Dedding for useful discussions regarding brighttime measurements. 


\section{REFERENCES}

(1) Sauer, M.; Hofkens, J.; Enderlein, J. Handbook of Fluorescence Spectroscopy and Imaging: From Single Molecules to Ensembles; WileyVCH Verlag GmbH \& Co. KGaA: Weinheim, Germany, 2011.

(2) Hinterdorfer, P.; Oijen, A., Eds. Handbook of Single-Molecule Biophysics; Springer US: New York, NY, 2009.

(3) Elenko, M. P.; Szostak, J. W.; van Oijen, A. M. Single-Molecule Imaging of an in Vitro-Evolved RNA Aptamer Reveals Homogeneous Ligand Binding Kinetics. J. Am. Chem. Soc. 2009, 131, 9866-9867.

(4) van Oijen, A. M. Single-molecule approaches to characterizing kinetics of biomolecular interactions. Curr. Opin. Biotechnol. 2011, 22, $75-80$.

(5) Lu, H. P.; Xun, L.; Xie, X. S. Single-Molecule Enzymatic Dynamics. Science 1998, 282, 1877-1882.

(6) $\mathrm{Lu}, \mathrm{H}$. P. Sizing up single-molecule enzymatic conformational dynamics. Chem. Soc. Rev. 2014, 43, 1118-1143.

(7) Zheng, D.; Lu, H. P. Single-Molecule Enzymatic Conformational Dynamics: Spilling Out the Product Molecules. J. Phys. Chem. B 2014, 118, 9128-9140.

(8) McKinney, S. A.; Joo, C.; Ha, T. Analysis of Single-Molecule FRET Trajectories Using Hidden Markov Modeling. Biophys. J. 2006, 91, 1941-1951.

(9) Hellenkamp, B.; Schmid, S.; Doroshenko, O.; Opanasyuk, O.; Kühnemuth, R.; Rezaei Adariani, S.; Ambrose, B.; Aznauryan, M.; Barth, A.; Birkedal, V.; et al. Precision and accuracy of single-molecule FRET measurementsa multi-laboratory benchmark study. Nat. Methods 2018, 15, 669-676.

(10) Joo, C.; Ha, T. Single-Molecule FRET with Total Internal Reflection Microscopy. Cold Spring Harbor Protocols 2012, 2012, pdb.top072058.

(11) Rogers, M. L.; Boutelle, M. G. Real-Time Clinical Monitoring of Biomolecules. Annu. Rev. Anal. Chem. 2013, 6, 427-453.

(12) Fabrizio, E. D.; Schlücker, S.; Wenger, J.; Regmi, R.; Rigneault, H.; Calafiore, G.; West, M.; Cabrini, S.; Fleischer, M.; Hulst, N. F. v.; et al. Roadmap on biosensing and photonics with advanced nanooptical methods. J. Opt. 2016, 18, 063003.

(13) Taylor, A. B.; Zijlstra, P. Single-Molecule Plasmon Sensing: Current Status and Future Prospects. ACS Sensors 2017, 2, 11031122.

(14) Ameur, A.; Kloosterman, W. P.; Hestand, M. S. SingleMolecule Sequencing: Towards Clinical Applications. Trends Biotechnol. 2019, 37, 72-85.

(15) Moerner, W. E.; Fromm, D. P. Methods of single-molecule fluorescence spectroscopy and microscopy. Rev. Sci. Instrum. 2003, 74, 3597-3619.

(16) Klehs, K.; Spahn, C.; Endesfelder, U.; Lee, S. F.; Fürstenberg, A.; Heilemann, M. Increasing the Brightness of Cyanine Fluorophores for Single-Molecule and Superresolution Imaging. ChemPhysChem 2014, 15, 637-641.

(17) Tian, Y.; Halle, J.; Wojdyr, M.; Sahoo, D.; Scheblykin, I. G. Quantitative measurement of fluorescence brightness of single molecules. Methods Appl. Fluoresc. 2014, 2, 035003.

(18) Turunen, P.; Rowan, A. E.; Blank, K. Single-enzyme kinetics with fluorogenic substrates: lessons learnt and future directions. FEBS Lett. 2014, 588, 3553-3563.

(19) Drexhage, K. H. Influence of a dielectric interface on fluorescence decay time. J. Lumin. 1970, 1-2, 693-701.

(20) Khatua, S.; Orrit, M. Probing, Sensing, and Fluorescence Enhancement with Single Gold Nanorods. J. Phys. Chem. Lett. 2014, 5, 3000-3006.

(21) Busson, M. P.; Rolly, B.; Stout, B.; Bonod, N.; Bidault, S. Accelerated single photon emission from dye molecule-driven nanoantennas assembled on DNA. Nat. Commun. 2012, 3, 962.

(22) Kinkhabwala, A.; Yu, Z.; Fan, S.; Avlasevich, Y.; Müllen, K.; Moerner, W. E. Large single-molecule fluorescence enhancements produced by a bowtie nanoantenna. Nat. Photonics 2009, 3, 654-657.

(23) Regmi, R.; Al Balushi, A. A.; Rigneault, H.; Gordon, R.; Wenger, J. Nanoscale volume confinement and fluorescence enhancement with double nanohole aperture. Sci. Rep. 2015, 5, 15852.
(24) Flauraud, V.; Regmi, R.; Winkler, P. M.; Alexander, D. T. L.; Rigneault, H.; van Hulst, N. F.; García-Parajo, M. F.; Wenger, J.; Brugger, J. In-Plane Plasmonic Antenna Arrays with Surface Nanogaps for Giant Fluorescence Enhancement. Nano Lett. 2017, 17, 17031710.

(25) Yuan, H.; Khatua, S.; Zijlstra, P.; Yorulmaz, M.; Orrit, M. Thousand-fold Enhancement of Single-Molecule Fluorescence Near a Single Gold Nanorod. Angew. Chem., Int. Ed. 2013, 52, 1217-1221.

(26) Khatua, S.; Paulo, P. M. R.; Yuan, H.; Gupta, A.; Zijlstra, P.; Orrit, M. Resonant Plasmonic Enhancement of Single-Molecule Fluorescence by Individual Gold Nanorods. ACS Nano 2014, 8, 4440-4449.

(27) Puchkova, A.; Vietz, C.; Pibiri, E.; Wünsch, B.; Sanz Paz, M.; Acuna, G. P.; Tinnefeld, P. DNA Origami Nanoantennas with over 5000-fold Fluorescence Enhancement and Single-Molecule Detection at 25 M. Nano Lett. 2015, 15, 8354-8359.

(28) Laurence, T. A.; Ly, S.; Bourguet, F.; Fischer, N. O.; Coleman, M. A. Fluorescence Correlation Spectroscopy at Micromolar Concentrations without Optical Nanoconfinement. J. Phys. Chem. B 2014, 118, 9662-9667.

(29) Punj, D.; de Torres, J.; Rigneault, H.; Wenger, J. Gold nanoparticles for enhanced single molecule fluorescence analysis at micromolar concentration. Opt. Express 2013, 21, 27338-27343.

(30) Punj, D.; Mivelle, M.; Moparthi, S. B.; van Zanten, T. S.; Rigneault, H.; van Hulst, N. F.; García-Parajó, M. F.; Wenger, J. A plasmonic /'antenna-in-box/' platform for enhanced single-molecule analysis at micromolar concentrations. Nat. Nanotechnol. 2013, 8, 512-516.

(31) Punj, D.; Regmi, R.; Devilez, A.; Plauchu, R.; Moparthi, S. B.; Stout, B.; Bonod, N.; Rigneault, H.; Wenger, J. Self-Assembled Nanoparticle Dimer Antennas for Plasmonic-Enhanced SingleMolecule Fluorescence Detection at Micromolar Concentrations. ACS Photonics 2015, 2, 1099-1107.

(32) Wenger, J. Fluorescence Enhancement Factors on Optical Antennas: Enlarging the Experimental Values without Changing the Antenna Design. Int. J. Opt. 2012, 2012, No. 828121.

(33) Wenger, J. Fluorescence spectroscopy enhancement on photonic nanoantennas. arXiv (physics) 2017, 1709.06749. https:// arxiv.org/abs/1709.06749.

(34) Visser, E. W.; Horáček, M.; Zijlstra, P. Plasmon Rulers as a Probe for Real-Time Microsecond Conformational Dynamics of Single Molecules. Nano Lett. 2018, 18, 7927-7934.

(35) Schuler, B.; Eaton, W. A. Protein folding studied by singlemolecule FRET. Curr. Opin. Struct. Biol. 2008, 18, 16-26.

(36) Borgia, M. B.; Borgia, A.; Best, R. B.; Steward, A.; Nettels, D.; Wunderlich, B.; Schuler, B.; Clarke, J. Single-molecule fluorescence reveals sequence-specific misfolding in multidomain proteins. Nature 2011, 474, 662-665.

(37) Dobson, C. M. Protein folding and misfolding. Nature 2003, 426, 884-890.

(38) Wenger, J.; Gérard, D.; Dintinger, J.; Mahboub, O.; Bonod, N.; Popov, E.; Ebbesen, T. W.; Rigneault, H. Emission and excitation contributions to enhanced single molecule fluorescence by gold nanometric apertures. Opt. Express 2008, 16, 3008.

(39) Gérard, D.; Wenger, J.; Bonod, N.; Popov, E.; Rigneault, H.; Mahdavi, F.; Blair, S.; Dintinger, J.; Ebbesen, T. W. Nanoapertureenhanced fluorescence: Towards higher detection rates with plasmonic metals. Phys. Rev. B: Condens. Matter Mater. Phys. 2008, $77,045413$.

(40) Wientjes, E.; Renger, J.; Cogdell, R.; van Hulst, N. F. Pushing the Photon Limit: Nanoantennas Increase Maximal Photon Stream and Total Photon Number. J. Phys. Chem. Lett. 2016, 7, 1604-1609.

(41) Hohenester, U.; Trügler, A. MNPBEM A Matlab toolbox for the simulation of plasmonic nanoparticles. Comput. Phys. Commun. 2012, 183, 370-381.

(42) Beuwer, M. A.; Prins, M. W. J.; Zijlstra, P. Stochastic Protein Interactions Monitored by Hundreds of Single-Molecule Plasmonic Biosensors. Nano Lett. 2015, 15 (5), 3507-3511. 
(43) Jungmann, R.; Steinhauer, C.; Scheible, M.; Kuzyk, A.; Tinnefeld, P.; Simmel, F. C. Single-Molecule Kinetics and SuperResolution Microscopy by Fluorescence Imaging of Transient Binding on DNA Origami. Nano Lett. 2010, 10, 4756-4761.

(44) Schnitzbauer, J.; Strauss, M. T.; Schlichthaerle, T.; Schueder, F.; Jungmann, R. Super-resolution microscopy with DNA-PAINT. Nat. Protoc. 2017, 12, 1198-1228.

(45) Vogelsang, J.; Kasper, R.; Steinhauer, C.; Person, B.; Heilemann, M.; Sauer, M.; Tinnefeld, P. A Reducing and Oxidizing System Minimizes Photobleaching and Blinking of Fluorescent Dyes. Angew. Chem., Int. Ed. 2008, 47, 5465-5469.

(46) Eggeling, C.; Volkmer, A.; Seidel, C. A. M. Molecular Photobleaching Kinetics of Rhodamine 6G by One- and Two-Photon Induced Confocal Fluorescence Microscopy. ChemPhysChem 2005, 6, 791-804.

(47) Zhang, Y.; Aslan, K.; Previte, M. J. R.; Malyn, S. N.; Geddes, C. D. Metal-Enhanced Phosphorescence: Interpretation in Terms of Triplet-Coupled Radiating Plasmons. J. Phys. Chem. B 2006, 110, 25108-25114.

(48) Stefani, F. D.; Vasilev, K.; Bocchio, N.; Gaul, F.; Pomozzi, A.; Kreiter, M. Photonic mode density effects on single-molecule fluorescence blinking. New J. Phys. 2007, 9, 21-21.

(49) Chaudhuri, D.; Li, D.; Sigmund, E.; Wettach, H.; Höger, S.; Lupton, J. M. Plasmonic surface enhancement of dual fluorescence and phosphorescence emission from organic semiconductors: effect of exchange gap and spin-orbit coupling. Chem. Commun. 2012, 48, 6675.

(50) Gill, R.; Tian, L.; van Amerongen, H.; Subramaniam, V. Emission enhancement and lifetime modification of phosphorescence on silver nanoparticle aggregates. Phys. Chem. Chem. Phys. 2013, 15, 15734

(51) Wenger, J.; Cluzel, B.; Dintinger, J.; Bonod, N.; Fehrembach, A.-L.; Popov, E.; Lenne, P.-F.; Ebbesen, T. W.; Rigneault, H. Radiative and Nonradiative Photokinetics Alteration Inside a Single Metallic Nanometric Aperture. J. Phys. Chem. C 2007, 111, 1146911474.

(52) Eggeling, C.; Widengren, J.; Rigler, R.; Seidel, C. A. M. Photobleaching of Fluorescent Dyes under Conditions Used for Single-Molecule Detection: Evidence of Two-Step Photolysis. Anal. Chem. 1998, 70, 2651-2659.

(53) Lakowicz, J. R. Principles of Fluorescence Spectroscopy, 3rd ed.; Springer: New York, 2006. 